\title{
REFLEXIVE TENSOR PRODUCTS AND SPACES OF OPERATORS
}

\section{KENNETH L. POTHOVEN}

ABSTRACT. By establishing conditions for which certain tensor products of Banach spaces are reflexive, conditions are given for which spaces of $k$-nuclear operators, absolutely $k$-summing operators, quasi $k \cdot$ nuclear operators, and $k$-integral operators are reflexive.

Let $X$ and $Y$ denote real or complex Banach spaces, and let $\left(x_{i}\right)_{i \in I}$ denote a sequence of elements in $X$. For $1 \leq k<\infty$, define $N_{k}\left(\left(x_{i}\right)_{i \in I}\right)$ or $N_{k}\left(x_{i}\right)$ by

$$
N_{k}\left(x_{i}\right)=\left(\sum_{i \in I}\left|x_{i}\right|^{k}\right)^{1 / k},
$$

and define $N_{\infty}\left(x_{i}\right)$ by the equation

$$
N_{\infty}\left(x_{i}\right)=\sup _{i \in I}\left|x_{i}\right|
$$

For $1 \leq k \leq \infty$, define $M_{k}\left(x_{i}\right)$ by the expression

$$
M_{k}\left(x_{i}\right)=\sup _{\left|x^{*}\right| \leq 1} N_{k}\left(x^{*}\left(x_{i}\right)\right) \text {, }
$$

where $x^{*} \in X^{*}$, the dual of $X$.

Let $X \otimes Y$ represent the algebraic tensor product of $X$ and $Y$, and for $u \in X \otimes Y$ define $d_{k}(u)$ for $1 \leq k \leq \infty$ by the equation

$$
d_{k}(u)=\inf \left\{M_{k^{\prime}}\left(x_{i}\right) N_{k}\left(y_{i}\right)\right\}
$$

where $u=\sum_{i=1}^{n} x_{i} \otimes y_{i}$, the infimum is taken over all such representations of $u, k^{\prime}=k / k-1$ if $k \neq 1, k^{\prime}=\infty$ if $k=1$, and $k^{\prime}=1$ if $k=\infty$. It can be

Received by the editors June 4, 1972 and, in revised form, June 5, 1973. AMS (MOS) subject classifications (1970). Primary 46B10; Secondary 47B10, 47D15, 46M05.

Key words and phrases. Tensor products of Banach spaces, reflexive, absolutely $k$-summing operators, $k$-nuclear operators, $k$-integral operators, quasi $k$-nuclear operators. 
verified (see [6]) that $d_{k}$ for $1 \leq k \leq \infty$ defines a norm on $X \otimes Y$ with $d_{k}(x \otimes y)=|x||y|$ for $x \in X$ and $y \in Y$. Denote by $X \otimes_{d_{k}} Y$ the space $X \otimes Y$ with norm $d_{k}$, and by $X \hat{\otimes}_{d_{k}} Y$ the completion of $X \otimes_{d_{k}} Y$.

If $x^{*} \in X^{*}$ and $y^{*} \in Y^{*}, x^{*} \otimes y^{*}$ in $X^{*} \otimes Y^{*}$ can be considered as the scalar-valued linear operator on $X \otimes Y$ defined by

$$
x^{*} \otimes y^{*}\left(\sum_{i=1}^{n} x_{i} \otimes y_{i}\right)=\sum_{i=1}^{n} x^{*}\left(x_{i}\right) y^{*}\left(y_{i}\right) .
$$

For a fixed expression $u=\sum_{i=1}^{m} x_{i}^{*} \otimes y_{i}^{*}$ in $X^{*} \otimes Y^{*}$, define $d_{k}^{\prime}(u)$ for $1 \leq k \leq \infty$ as the least constant $C$ (finite or infinite) satisfying the inequality

$$
\left|\sum_{i=1}^{m} x_{i}^{*} \otimes y_{i}^{*}\left(\sum_{i=1}^{n} x_{i} \otimes y_{i}\right)\right| \leq C d_{k}\left(\sum_{i=1}^{n} x_{i} \otimes y_{i}\right)
$$

for all expressions $\sum_{i=1}^{n} x_{i} \otimes y_{i}$ in $X \otimes_{d_{k}} Y$. It can be shown that $d_{k}^{\prime}$ defines a norm on $X^{*} \otimes Y^{*}$ with $d_{k}^{\prime}\left(x^{*} \otimes y^{*}\right)^{k}=\left|x^{*}\right|\left|y^{*}\right| \cdot d_{k}^{0}$ is called the associate norm of $d_{k}$, and clearly $X^{*} \otimes_{d_{k}^{\prime}} Y^{*}$ is a normed linear subspace of $\left(X \hat{\otimes}_{d_{k}} Y\right)^{*}$.

In a similar fashion, an element $x \otimes y$ (hence an element $\sum_{i=1}^{n} x_{i} \otimes y_{i}$ ) in $X \otimes Y$ can be considered as a scalar-valued operator on $X^{*} \otimes Y^{*}$ by means of the equation

$$
x \otimes y\left(\sum_{i=1}^{m} x_{i}^{*} \otimes y_{i}^{*}\right)=\sum_{i=1}^{m} x_{i}^{*}(x) y_{i}^{*}(y)
$$

Denote by $\widetilde{d}_{k}(u)$ the "norm" of an element $u=\sum_{i=1}^{n} x_{i} \otimes y_{i}$ in $X \otimes Y$ considered as an element of $\left(X^{*} \hat{\otimes}_{d_{k}^{\prime}} Y^{*}\right)^{*}$. It can also be verified that $\widetilde{d}_{k}$ defines a norm on $X \otimes Y$ and $\widetilde{d}_{k}(u) \leq d_{k}(u)$ for $u \in X \otimes Y$. Moreover if $X$ or $Y$ satisfies the metric approximation property (m.a.p.) (see [1, pp. 164-181]), then $\tilde{d}_{k}(u)=d_{k}(u)$ for $u \in X \otimes Y$ (see [6]).

It should be noted that $d_{1}=\pi$, the greatest crossnorm of Schatten [5].

The following facts, whose proofs can be found in [6], show the relationships between $X \hat{\otimes}_{d_{k}} Y$ and certain spaces of operators.

(1) $\left(X \hat{\otimes}_{d_{k}} Y\right)^{*}$ is isometrically isomorphic to $S^{k}\left(X, Y^{*}\right)$, the space of absolutely $k^{\prime}$-summing operators from $X$ to $Y^{*}$ (see [6]).

(2) $Y \hat{\otimes}_{d_{k}} X^{*}$ is isometrically isomorphic to $N_{k}(X, Y)$ if $Y$ or $X^{*}$ satisfies the approximation property (a.p.), where $N_{k}(X, Y)$ denotes the space of $k$-nuclear operators from $X$ to $Y$. 
(3) $X^{*} \hat{\otimes}_{d_{k}^{\prime}} Y$ is isometrically isomorphic to $Q N_{k}(X, Y)$, the space of quasi $k^{\prime}$-nuclear operators from $X$ to $Y$, if $X^{*}$ or $Y$ satisfies the m.a.p.

(4) $\left(X^{*} \hat{\otimes}_{d_{k}^{\prime}} Y\right)^{*}$ is isometrically isomorphic to $I_{k}\left(Y, X^{* *}\right)$, the space of $k$-integral operators from $Y$ to $X^{* *}$.

Lemma 1. Let $X$ and $Y$ be Banach spaces with $X$ or $Y$ satisfying the m.a.p. The following statements are equivalent.

(a) $X \hat{\otimes}_{d_{k}} Y$ is reflexive.

(b) $X^{*} \hat{\otimes}_{d_{k}^{\prime}} Y^{*}$ is reflexive.

(c) $\left(X \hat{\otimes}_{d_{k}} Y\right)^{*}=X^{*} \hat{\otimes}_{d_{k}^{\prime}} Y^{*}$ and $\left(X^{*} \hat{\otimes}_{d_{k}^{\prime}} Y^{*}\right)^{*}=X \hat{\otimes}_{d_{k}} Y$ (isometrically).

Proof. Since $X^{*} \hat{\otimes}_{d_{k}^{\prime}} Y^{*}$ is a subspace of $\left(X \hat{\otimes}_{d_{k}} Y\right)^{*}$, statement (a) trivially implies statement (b). Now suppose statement (b) holds. Since $\widetilde{d}_{k}=d_{k}$ when $X$ or $Y$ satisfies the m.a.p., $X \hat{\otimes}_{d_{k}} Y$ can be isometrically embedded into $\left(X^{*} \hat{\otimes}_{d_{k}^{\prime}} Y^{*}\right)^{*}$ by a mapping $h$. Hence with $i$ and $j$ representing the obvious isometries, and $b^{*}$ denoting the adjoint of $h$, the following diagram commutes.

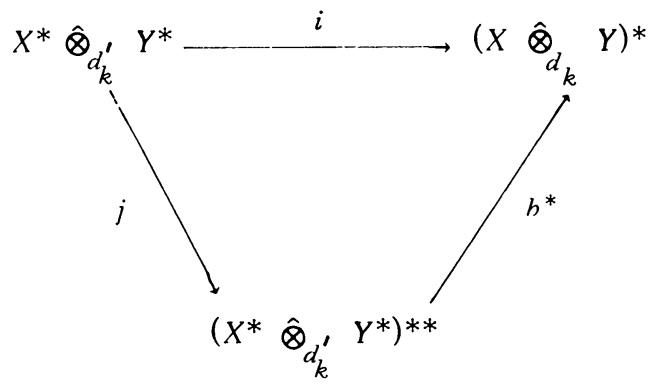

For each $u \in\left(X \hat{\otimes}_{d_{k}} Y\right)^{*}$, there exists an element $v$ in $\left(X^{*} \hat{\otimes}_{d_{k}^{\prime}} Y^{*}\right)^{* *}$ such that $h^{*}(v)=u$ and $|v|=|u|$. Thus if $X^{*} \hat{\otimes}_{d_{k}^{\prime}} Y^{*}$ is reflexive, then $j$ is an isometric isomorphism. This means both $i$ and $h$ are isomorphisms. Hence

$$
X^{*} \hat{\otimes}_{d_{k}^{\prime}} Y^{*}=\left(X \hat{\otimes}_{d_{k}} Y\right)^{*} \text { and }\left(X^{*} \hat{\otimes}_{d_{k}^{\prime}} Y^{*}\right)^{*}=X \hat{\otimes}_{d_{k}} Y
$$

Note that if $X^{*} \hat{\otimes}_{d_{k}^{\prime}} Y^{*}$ is reflexive, then $j$ is an isometric isomorphism and, hence, $i$ must be an isometric isomorphism. Hence $\left(X \hat{\otimes}_{d_{k}} Y\right)^{*}$ is reflexive, which implies that $X \hat{\otimes}_{d_{k}} Y$ is reflexive.

Finally, by taking the duals on both sides of the first equation in (c) and using the second equation, statement (c) is seen to imply (a). This concludes the proof.

Note that if $X$ and $Y$ are reflexive Banach spaces and $X$ or $Y$ satisfies 
the a.p., then using the facts stated above, condition (c) of the proposition becomes

(*) $S^{k^{\prime}}\left(X, Y^{*}\right)=Q N_{k}{ }^{\prime}\left(X, Y^{*}\right)$ and $N_{k}\left(Y^{*}, X\right)=I_{k}\left(Y^{*}, X\right)$ (isometrically). However, Persson in [3] has proved that, for $1 \leq k<\infty$, if $X$ is reflexive then

$(* *) S^{k}(X, Y)=Q N_{k}(X, Y)$ and $N_{k}(X, Y)=I_{k}(X, Y)$ (isometrically) for any Banach space $Y$. Therefore with this information and the above proposition, one obtains

Proposition 2. If $X \neq 0$ and $Y \neq 0$ are Banach spaces with $X$ or $Y$ satisfying the m.a.p., then the following are equivalent for $1<k<\infty$.

(a) $X$ and $Y$ are reflexive.

(b) $X \hat{\otimes}_{d_{k}} Y$ is reflexive.

(c) $S^{k}(X, Y)$ is reflexive.

(d) $Q N_{k}(X, Y)$ is reflexive.

(e) $I_{k}(X, Y)$ is reflexive.

(f) $N_{k}(X, Y)$ is reflexive.

Proof. Since $X$ and $Y$ are subspaces of each of the spaces in parts (b) through ( $f$ ), each of the statements (b) through (f) implies (a). Now suppose (a) is true. Then (b) is true by condition $(*)$ and Lemma 1. (c) is true since $S^{k}(X, Y)$ is the dual of $X \otimes_{d_{k^{\prime}}} Y^{*}$. (d) is then true by $(* *)$. (f) is true by fact (2) and, hence, (e) is true by $(* *)$.

In the case $d_{k}=\pi$ (when $k=1$ ), a different result is obtained. $S^{\infty}\left(X, Y^{* *}\right)=B\left(X, Y^{* *}\right)$, the space of all bounded linear operators from $X$ to $Y^{* *}$, and $Q N_{\infty}\left(X, Y^{* *}\right)=K\left(X, Y^{* *}\right)$, the space of compact operators from $X$ to $Y^{* *}$ (see [4, p. 56]) provided $X^{*}$ or $Y^{* *}$ have the a.p. Also when $Y$ is reflexive, $N_{1}\left(Y^{* *}, X\right)=I_{1}\left(Y^{* *}, X\right)$. Hence, using Lemma 1 , one obtains

Proposition 3. Let $X$ and $Y$ be reflexive Banach spaces with $X$ or $Y$ satisfying the m.a.p. Then $B(X, Y)$ is reflexive if and only if each operator $T: X \rightarrow Y$ is compact (see also [2]).

From the case when $k=+\infty$, since $S^{1}(X, Y)=Q N_{1}(X, Y)$ when $X$ is reflexive, condition $(*)$ indicates

Proposition 4. If $X$ and $Y$ are reflexive Banach spaces with $X$ or $Y$ satisfying the m.a.p. then the following statements are equivalent.

(a) $X \hat{\otimes}_{d_{\infty}} Y$ is reflexive.

(b) $N_{\infty}\left(Y^{*}, X\right)=I_{\infty}\left(Y^{*}, X\right)$.

(c) $S^{1}\left(X, Y^{*}\right)$ is reflexive. 
(d) $Q N_{1}\left(X, Y^{*}\right)$ is reflexive.

(e) $N_{\infty}\left(Y^{*}, X\right)$ is reflexive.

(f) $I_{\infty}\left(Y^{*}, X\right)$ is reflexive.

In [4, p. 57] it is verified that $N_{\infty}\left(H_{1}, H_{2}\right)=I_{\infty}\left(H_{1}, H_{2}\right)$ if $H_{1}$ and $H_{2}$ are Hilbert spaces. However $X \hat{\otimes}_{d_{\infty}} Y$ is reflexive for $X$ and $Y$ other than Hilbert spaces. Since

(a) $d_{\infty}$ is equivalent to $d_{k}$ for $2 \leq k \leq \infty$ on $H \otimes F$, where $H$ is a Hilbert space and $F$ is an arbitrary Banach space (see $[6$, p. 95]), and

(b) $d_{k}$ is equivalent to $d_{\infty}$ for $2 \leq k \leq \infty$ on $L^{p}(\mu) \otimes F$ for $1 \leq p \leq 2$, where $\mu$ is a (Radon) measure on a locally compact space and $F$ is any Banach space (see [6, p. 97]),

any one of the equivalent statements of Proposition 4 holds when $X$ is a Hilbert space or a space $L^{p}(\mu)$ for $1<p \leq 2$ and $Y$ is any reflexive space.

\section{REFERENCES}

1. A. Grothendieck, Produits tensoriels topologiques et espaces nucléaires, Mem. Amer. Math. Soc. No. 16 (1955). MR 17, 763.

2. J. R. Holub, Hilbertian operators and reflexive tensor products, Pacific J. Math. 36 (1971), 185-194. MR 46 \#645.

3. A. Persson, On some properties of p-nuclear und p-integral operators, Studia Math. 33 (1969), 213-222. MR 40 \#769.

4. A. Persson and A. Pietsch, p-nukleare und p-integrale Abbildungen in Banachräumen, Studia Math. 33 (1969), 19-62. MR 39 \#4645.

5. R. Schatten, A theory of cross-spaces, Ann. of Math. Studies, no. 26, Princeton Univ. Press, Princeton, N. J., 1950. MR 12, 186.

6. P. Saphar, Produits tensoriels d'espaces de Banach, et classes d'applications linéaires, Proc. Internat. Colloq. on Nuclear Spaces and Ideals in Operator Algebras (Warsaw, 1969), Studia Math. 38 (1970), 71-100. MR 43 \#878.

DEPARTMENT OF MATHEMATICS, UNIVERSITY OF SOUTH FLORIDA, TAMPA, FLORIDA 33620 